used to evaluate $\triangle \mathrm{CDAI}$. Subgroup analyses were conducted by line of therapy, after excluding switch pts.

Results: Overall, 3959 pts met inclusion criteria and 2045 had data on PPF; 344 and 814 pts received ABA and anti-TNF. Mean (SD) age of ABA pts was 60.3 (12.5) yrs and median (interquartile range; IQR) CDAI was 25.5 (18.1). Mean (SD) age of anti-TNF pts was 56.4 (13.3) yrs and median CDAI (IQR) was 19.8 (18.1). PPF+ pts treated with ABA (vs all other ABA pts) had a better CDAl outcome at $6 \mathrm{M}(-9.7$ [16.1] vs -7.5 [14.4]). In sensitivity analyses, the difference persisted $(-10.4$ vs $-8.4 ; \mathrm{Fig})$. PPF + pts treated with $A B A$ as first-line therapy (vs all other ABA pts) had a better CDAl outcome $(-13.4$ vs -10.3$)$. Similar trends were not observed in the anti-TNF cohort (Fig). Adjusted mean (SE) $\triangle \mathrm{CDAl}$ in PPF+ vs PPF- pts treated with ABA was -12.1 (2.01) vs -9.5 (0.79); covariates included in the model were age, sex and BL CDAI $(p=0.24)$. During follow-up, more PPF+ pts treated with ABA changed from high/medium to LDA or remission vs all other pts ( $34 \%$ vs $25 \%$ ), while this was not seen in anti-TNF pts ( 45 vs $42 \%$ ).

Conclusions: Pts with RA treated with abatacept had higher disease activity at $\mathrm{BL}$ and a greater reduction in disease activity was observed in seropositive pts with RA and erosions compared with all other abatacept pts. Similar trends were not observed in anti-TNF pts. No direct comparisons between treatments were conducted in these cohorts.

[1] Humphreys JH, et al. Arthritis Res Ther 2014;16:483.

[2] Quinn MA, et al. Rheumatology (Oxford) 2006;45:478-80.

[3] Dixey J, et al. J Rheumatol Suppl 2004;69:48-54.

Disclosure of Interest: E. Alemao Shareholder of: Bristol-Myers Squibb, Employee of: Bristol-Myers Squibb, K. Knapp Employee of: Discus Analytics, V. Anupindi: None declared, S. Annamalai: None declared, G. Craig Shareholder of: Discus Analytics, Grant/research support from: Bristol-Myers Squibb, Consultant for: Premera Blue Cross/Blue Shield of Washington and Alaska, Employee of: Arthritis Northwest, Speakers bureau: Bristol-Myers Squibb, UCB, Genentech, Celgene, Novartis

DOI: 10.1136/annrheumdis-2017-eular.1517

\section{FRI0233 THE RETENTION RATES OF ABATACEPT IN ELDERLY RA PATIENTS (65 YEARS AND ABOVE) WHO CANNOT BE TREATED WITH METHOTREXATE: COMPARISON WITH ETANERCEPT AND TOCILIZUMAB; A SINGLE-CENTER RETROSPECTIVE, CASE-CONTROL STUDY}

E. Kikuchi, N. Aoki, T. Yoshioka, T. Okai. Center for Rheumatology and Joint Surgery, Kawakita General Hospital, Tokyo, Japan

Background: There are many elderly rheumatoid arthritis (RA) patients who cannot be treated with methotrexate (MTX) for many reasons, but data about the therapeutic strategies by biologic agents for the patients are insufficient.

Objectives: To analyze the retention rate of abatacept in elderly patients with RA who cannot be treated with MTX.

Methods: Data were retrospectively collected from the medical records of patients with rheumatoid arthritis at our center. Abatacept (ABT), etanercept (ETN), or tocilizumab (TCZ) was administered to 68 elderly RA patients who could not be treated with MTX. We analyzed the retention rate of each group by Kaplan-Meier curves and the log-rank test. The primary end point was the 24-month retention rate of the biologics without discontinuation due to adverse events, loss or primary lack of effectiveness.

Results: In the ABT group ( 26 cases: a mean age of $77.8 \pm 6.3$ years, ACPA positive $92.3 \%$, oral steroid use $34.6 \%$ ), the cumulative retention rates for both 12 and 24 months were 0.699 . In the ETN group (26 cases: a mean age of $75.8 \pm 5.1$ years, ACPA positive $92.0 \%$, oral steroid use $73.1 \%$ ), the cumulative retention rates for 12 and 24 months were 0.450 and 0.315 , respectively. In the TCZ group (16 cases: a mean age of $73.7 \pm 5.6$ years, ACPA positive $87.5 \%$, oral steroid use $56.3 \%$ ), the cumulative retention rates for 12 and 24 months were 0.433 and 0.325 , respectively.

There was a significant difference in the retention rates between $A B T$ groups and the other two groups [log-rank test, $p=0.018$ (ABT vs. ETN), 0.047 (ABT vs.

Kaplan-Meier drug survival estimates for
abatacept (ABT), etanercept (ETN), Tociliz

in elderly RA patients ( $\geq 65$ years) who cannot be treated with methotrexate

(endpoint: discontinuation due to adverse events, loss or primary lack of effectiveness)

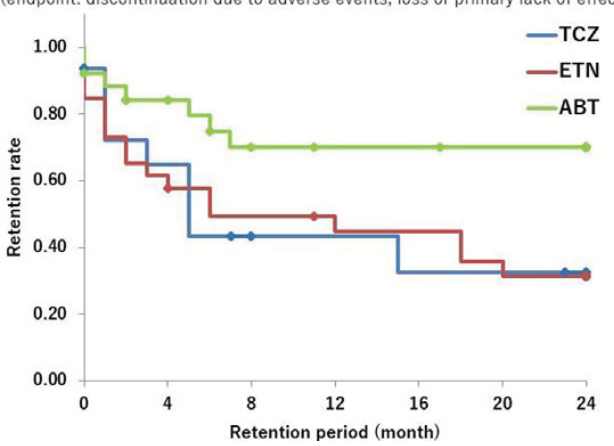

TCZ)]. There are no significant retention rates between ETN groups and TCZ groups.

Three cases (11.5\%) in the ABT group were discontinued the biologic agents by hospitalization for severe infection within 24 months. Five cases $(19.2 \%)$ in the ETN group, six cases (37.5\%) in TCZ group were hospitalized for severe infection, respectively.

Conclusions: Our data suggested that abatacept can be used for a period longer than etanercept or tocilizumab for elderly RA patients who cannot be treated with methotrexate.

References:

[1] Harigai, et al. Mod Rheumatol. 2016 Jul 3; 26(4): 491-498.

Disclosure of Interest: None declared

DOI: 10.1136/annrheumdis-2017-eular.6045

\section{FRI0234 CHOICE OF BIOLOGIC THERAPY FOLLOWING RITUXIMAB: INFLUENCING FACTORS IN A FRENCH MULTICENTER COHORT OF RHEUMATOID ARTHRITIS}

G. Vial ${ }^{1}$, A. De Pouilly ${ }^{2}$, L. Scouppe ${ }^{3}$, B. Pereira ${ }^{4}$, C. Lukas ${ }^{5}$, C. Daien ${ }^{5}$, A. Ruyssen-Witrand ${ }^{6}$, P. Vergne-Salle ${ }^{2}, A$. Tournadre ${ }^{1}, C$. Richez ${ }^{3}$.

${ }^{1}$ Rheumatology, CHU, Clermont Ferrand; ${ }^{2}$ Rheumatology, CHU, LIMOGES;

${ }^{3}$ Rheumatology, CHU, Bordeaux; ${ }^{4} \mathrm{DRCl}, \mathrm{CHU}$, Clermont Ferrand;

${ }^{5}$ Rheumatology, $\mathrm{CHU}$, Montpellier; ${ }^{6}$ Rheumatology, $\mathrm{CHU}$, Toulouse, France

Background: In rheumatoid arthritis (RA), the long lasting effect of rituximab (RTX) on B lymphocyte depletion may influence the choice of biologic after RTX failure but there is currently no specific recommendation about the optimal strategy.

Objectives: We assessed factors which may have influenced the choice of biologic following failure to RTX in RA patients and analysed the effectiveness of these biologics.

Methods: A retrospective study of RA patients, who had started a new biologic during the year after RTX discontinuation, was conducted between 2009 and 2016 in 5 French rheumatology centers. We collected at baseline (at the time the biologic was introduced after RTX) and during follow-up ( 3,6 and 12 months), data about patients and disease. Characteristics of the patients receiving tocilizumab (TCZ), abatacept (ABA) or anti-TNF following RTX, the EULAR response and retention rate were compared using univariate and multivariate analyses.

Results: 152 RA patients (117 women, mean age $56.9 \pm 13$ years) were analysed. RA duration was 14.3 years [IQR 7.8-19.8], mean DAS28 ESR at baseline 4.8+1.3. 57 patients $(37.5 \%)$ received TCZ, $47(31 \%)$ anti-TNF and $48(31.2 \%)$ ABA following RTX. $87 \%$ had received anti-TNF prior to RTX. No significant difference in the biologics prescription profile was noted across centers. At baseline, sex, disease characteristics and activity, use of concomitant DMARDs or prednisone were not different between the 3 groups. There was no difference in the number of cycles or dose of RTX received and the number of previous anti-TNF treatment. Patients receiving ABA were slightly older ( 59.8 years vs 54 years for TCZ and 57.8 years for anti-TNF, $p=0.06$ ). Multimorbidity index (MMI) assessing the number of comorbidities was higher in the group ABA but not significantly (MMI count $2 \pm 1.7$ for ABA, $1.6 \pm 1.5$ for TCZ, $1.7 \pm 1.4$ for anti-TNF, $\mathrm{p}=0.5$ ). At 3,6 and 12 months, DAS28ESR was lower in patients with TCZ as compared to those with anti-TNF or ABA (Table) but tender and swollen joint counts did not differ. The EULAR good-or-moderate response rates were similar across groups (Table). After adjustment on age, sex, disease duration, MMI count,

Table 1. Change in DAS28-ESR score and EULAR response at 3, 6, 12 months according to the biologic following RTX treatment

\begin{tabular}{|c|c|c|c|c|}
\hline & $\operatorname{TCZ}(n=57)$ & Anti TNF $(n=47)$ & ABA $(n=48)$ & $\mathrm{p}$ \\
\hline \multicolumn{5}{|c|}{ DAS28 ESR (mean $\pm S D)$} \\
\hline M3 & $2,7 \pm 1,2$ & $3,5 \pm 1,7$ & $3,7 \pm 1,3$ & 0.002 \\
\hline M6 & $2,6 \pm 1,5$ & $3,5 \pm 1,7$ & $3,5 \pm 1,2$ & 0.01 \\
\hline M12 & $2,5 \pm 1,2$ & $3,1 \pm 1,7$ & $3,3 \pm 1,3$ & 0.04 \\
\hline \multicolumn{5}{|c|}{ EULAR Good-Moderate response $\mathrm{n}(\%)$} \\
\hline M3 & $29(76)$ & $12(63)$ & $17(52)$ & 0.09 \\
\hline M6 & $22(79)$ & $8(57)$ & $19(73)$ & 0.34 \\
\hline M12 & $21(75)$ & $7(70)$ & $16(73)$ & 0.95 \\
\hline
\end{tabular}

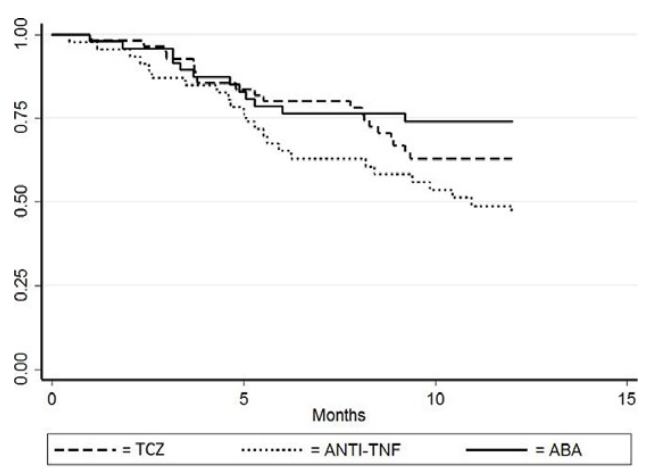

Figure 1: Retention rate of $A B A, T C Z$, antiTNF treatments after failure of $R T X$. 
number of previous anti-TNF, use of concomitant DMARDs and prednisone, drug retention rate at 1 year was significantly better for ABA compared to anti-TNF $(p=0.02)$ and for TCZ compared to anti-TNF $(p=0.04)$, but no difference was found between TCZ and ABA ( $p=0.62$ ) (Figure).

Conclusions: Patients who received ABA following failure of RTX, seem to be older and with more comorbidities. Similar rate of good-or-moderate EULAR response was observed between patients receiving TCZ, ABA or anti-TNF. However, drug retention rate was better with TCZ and ABA compared to anti-TNF after RTX discontinuation.

Disclosure of Interest: None declared

DOI: 10.1136/annrheumdis-2017-eular.5472

\section{FRI0235 CNCOMITANT USE OF INTRAVENOUS METHYLPREDNISOLONE TO OBTAIN HIGHER RETENTION RATE OF ABATACEPT IN RHEUMATOID ARTHRITIS}

H. Sawada, M. Suda, M. Kishimoto, M. Okada. Immuno-Rheumatology Center, St Luke's International Hospital, Tokyo, Japan

Background: Abatacept (ABT) is a widely used biologic for treating rheumatoid arthritis (RA).

Objectives: Concomitant use of intravenous corticosteroids with ABT infusion may contribute to achieve earlier remission and higher retention rates.

Methods: We conducted a retrospective cohort study at St. Luke's International Hospital, Tokyo, Japan, from January 2010 to June 2016. Patients who met the 2010 ACR/EULAR classification criteria for RA and treated with ABT were included in the study. We excluded patients who use two or more biologics prior to initiation of ABT. Our primary outcome was treatment retention rates of ABT at week 24. Secondary outcomes were changes in C-reactive protein (CRP) and erythrocyte sedimentation rate (ESR) during follow-up (week 0 vs. week 8). We compared these outcomes between the patients receiving concomitant intravenous methylprednisolone (mPSL) with first 3 consecutive doses of ABT and those not receiving it. Log-rank analysis and Fisher's exact test were applied for statistical analysis.

Results: 64 RA patients were included in the study. Mean age was $67.3( \pm 14.2)$ and $55(85.9 \%)$ were female. Among them, $13(20.3 \%)$ received concomitant ABT with mPSL. The dosage of $\mathrm{mPSL}$ ranged from $30 \mathrm{mg}$ to $250 \mathrm{mg}$ (median dosage was $40 \mathrm{mg}$ ). At week 24, the cumulative retention rates of the patients receiving $\mathrm{mPSL}$ (mPSL group) and those not receiving it (non-mPSL group) were $92.3 \%$ and $76.5 \%$, respectively. There was no significant difference in the retention rates between the two groups (log-rank test, $\mathrm{p}=0.21$ ) (figure). Changes in CRP between week 0 vs. week 8 were $-1.56 \mathrm{mg} / \mathrm{dl}( \pm 2.92)$ in $\mathrm{mPSL}$ group and $-1.08 \mathrm{mg} / \mathrm{dl}$ $( \pm 2.17)$ in non-mPSL group $(p=0.22)$, and those in ESR were $-8.23 \mathrm{~mm} / \mathrm{h}( \pm$ $18.40)$ in $\mathrm{mPSL}$ group and $-6.61 \mathrm{~mm} / \mathrm{h}( \pm 17.93)$ in non-mPSL group $(\mathrm{p}=0.75)$, respectively.

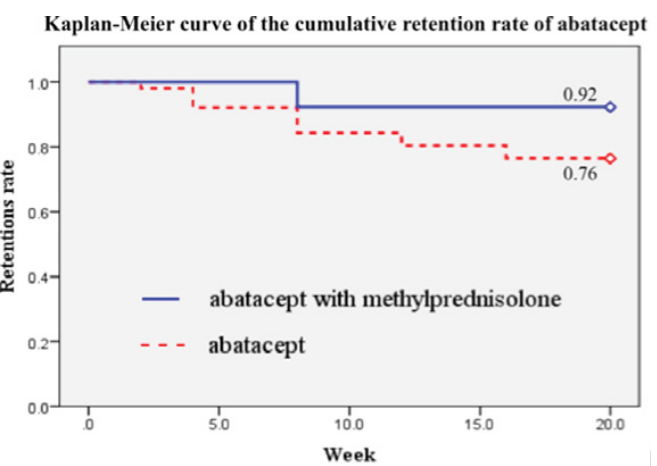

Conclusions: This is the first real world study to investigate whether ABT administrated with intravenous MPSL maintain higher retention rates in rheumatoid arthritis. Though there was no statistically significant difference, the retention rates of $A B T$ at 24 weeks were higher among patients receiving ABT and MPSL concomitantly, compared with $\mathrm{ABT}$ mono-therapy group. It may reflect rapid improvement of the disease activity.

Disclosure of Interest: None declared

DOI: 10.1136/annrheumdis-2017-eular.4716

\section{FRI0236 AFTER DISCONTINUATION OF THE 1ST TUMOR NECROSIS FACTOR INHIBITOR (TNFI), NON-TNFI BIOLOGIC AGENTS HAVE SIMILAR RESPONSES BUT HIGHER SURVIVAL COMPARED TO A 2ND COURSE OF A DIFFERENT TNFI: LONG-TERM PROSPECTIVE OBSERVATIONAL STUDY OF PATIENTS WITH RHEUMATOID ARTHRITIS IN A TERTIARY HOSPITAL OF GREECE}

I.D. Flouri ${ }^{1}$, A. Repa ${ }^{1}$, N. Avgoustidis ${ }^{1}$, N. Kougas ${ }^{1}$, A. Fanouriakis ${ }^{1}$ I. Papalopoulos ${ }^{1}$, C. Adamichou ${ }^{1}$, P. Kyfonidou ${ }^{1}$, E. Kampouraki ${ }^{1}$, M. Terizaki ${ }^{1}$, D.T. Boumpas ${ }^{2}$, G. Bertsias ${ }^{1}$, P. Sidiropoulos ${ }^{1} .{ }^{1}$ Rheumatology, Clinical
Immunology and Allergy, Faculty of Medicine-University of Crete, Heraklion, Crete; ${ }^{2} 4$ th Internal Medicine Department, Attikon University Hospital, Athens, Greece

Background: For rheumatoid arthritis (RA) patients who discontinue the first biologic agent (bDMARD), most commonly being a TNF inhibitor (TNFi), there is little evidence supporting the next best choice between a second TNFi course or a non-TNFi bDMARD in clinical practice.

Objectives: To compare the effectiveness and the adherence to therapy with non-TNFi versus TNFi administered as the second-line bDMARD in RA patients with one prior TNFi use.

Methods: All patients starting a bDMARD in the Rheumatology Department of the University Hospital of Heraklion, Crete, are included in a prospective observational study after their written informed consent. Data concerning disease activity at pre-specified time-points, drugs, comorbidities and any adverse events are recorded. For the present study we analyzed patients with RA starting their second course of a bDMARD after discontinuation of a TNFi. We compared DAS28 difference at 6 and 12 months using linear regression analysis and treatment retention using Kaplan-Meier survival curves with log-rank test.

Results: A total of 384 patients started a 2nd (different) TNFi [N=213 (Infliximab: 26, Adalimumab: 77, Etanercept: 89, Golimumab: 13 and Certolizumab: 8)] or a non-TNFi [N=171 (Rituximab:71, Abatacept:66, Tocilizumab:34)]. Patients' characteristics at baseline are described in the Table.

Two-year drug survival was higher for non-TNFi $(64 \%$ vs. $39 \%$, log rank $p<0.001)$ due to lower frequency of discontinuations for primary failure $(p<0.001)$ and adverse events $(\mathrm{p}=0.019)$

¿DAS28 was comparable between non-TNFi and TNFi patient groups both at 6 [mean (SD): -1.16 (1.29) and -1.07 (1.55) respectively, $\mathrm{p}=0.296$ ] and at 12 months $[-1.41(1.29)$ and $-1.39(1.26)$ respectively, $\mathrm{p}=0.670]$. In patients who did not receive co-therapy with methotrexate, significantly greater $\delta$ DAS28 was observed with a non-TNFi (-1.25 (1.29) vs.-0.68 (1.61), $\mathrm{p}=0.006)$. When the first TNFi was discontinued due to primary failure, we observed a trend for greater $\delta$ DAS28 in the non-TNFi patient group compared to the 2 nd TNFi group (-1.4 vs. $-1.0, p=0.12$ ) while the opposite was observed in patients who have experienced secondary failure to the $1 \mathrm{st}$ TNFi ( -0.81 vs $-1.48, p=0.18$ ), but this did not reach statistical significance, probably due to the low number of available patients.

Conclusions: In RA patients who need a 2nd bDMARD after discontinuation of a TNFi, administration of a non-TNFi results in similar clinical responses but higher treatment adherence compared to a second TNFi agent. In patients who do not receive methotrexate, responses are better with a non-TNFi bDMARD.

Disclosure of Interest: None declared

DOI: 10.1136/annrheumdis-2017-eular.6817

\section{FRI0237 SAFETY OF BIOSIMILAR INFLIXIMAB USE IN A MEDICAL DAY HOSPITAL: A CASE-SERIES}

J.M. Lopez Suarez ${ }^{1}$, J.M. Lopez Chozas ${ }^{2}$, E. Rubio Romero ${ }^{3}$. ${ }^{1}$ Family Doctor, CS Olivar de Quinto; ${ }^{2}$ Interne Medicine; ${ }^{3}$ Rheumatology, HU Virgen del Rocio, Sevilla, Spain

Background: The first biosimilar anti-NTF (infliximab) approved by the EMA (European Medicines Agency) began to be used throughout Europe in 2015. Since then, it has been widely used throughout the world, even replacing the original treatment approved in 1999 Remicade $^{\circledR}$ with Remsima ${ }^{\circledR}$, a biosimilar drug. This medicine is currently approved to treat people who have been diagnosed with rheumatoid arthritis, ankylosing spondylitis or psoriatic arthritis in the field of reheumatology, in addition to ulcerative colitis (UC) or Crohn's disease (EC) in gastroenterology. However, safety data of the use of Remsima ${ }^{\circledR}$ infliximab in a medical day hospital (MDH) are lacking.

Objectives: To report intrainfusion and postinfusion adverse events related to intravenous Remsima ${ }^{\circledR}$ use in a medical day hospital. Besides, we will describe the rehospitalizations and continuity of treatment in the follow up.

Methods: We designed a prospective uncontrolled case-series study. Patients referred from March 2015 to October 2016 to our MDH to receive intravenous Remsima ${ }^{\circledR}$ were consecutively enrolled. Demographic data were collected, and a harmonised active monitoring strategy was applied. We recorded the indication for treatment, the doses administered and the number and symptoms of acute transfusion reactions (ATR) occurring both in the infusion period and during the 3 hour postinfusion observation interval

Results: 2828 doses were administered, $0.67 \%$ presented ATR, all of them during the infusion and reported to the Regional Pharmacovigilance Center. $53 \%$ of those ATR were women and they were on average 35.78 years (SD 14.71). The mean dose used was $4,809 \mathrm{mg} / \mathrm{kg}$ (SD 0.47). The indications for treatment in patients suffering ATR were: EC (47\%), CU (26\%), Spondylitis $(21 \%)$ and Psoriasis $(5 \%)$. As a predominant symptom during ATR we found: shortness of breath $(36.89 \%)$, pruritus $(15.75 \%)$, flushing sensation $(15.75 \%)$, urticaria $(10.4 \%)$, alopecia $(5.2 \%)$, chest pain $(5.2 \%)$, paresthesia $(5.2 \%)$ and nausea (5.2\%). $95 \%$ of ATR showed complete recovery of the symptoms, $47 \%$ disappeared with the pause of infusion. However, they had prolonged in $\mathrm{MDH}$ stay, the infusion was stopped for 30 minutes and restarted at a lower rate according to the protocol. $21 \%$ of patients presenting ATR dropped out because of the symptomatology, which represents $0.14 \%$ of the total infusions.

Conclusions: Biosimilar Inflliximab seems to be safe in a MDH setting, given the 